\title{
Designing an ICT Solution for the Empowerment of Functional Independence of People with Mild Cognitive Impairment: Findings from Co-design Sessions with Older People
}

\author{
Silvia de los Ríos ${ }^{1(\bowtie)}$, Rebeca I. García-Betances ${ }^{1(\bowtie)}$, \\ Miguel Páramo', María Fernanda Cabrera-Umpiérrez ${ }^{1}$, \\ Marta Vancells ${ }^{2}$, Maite Garolera ${ }^{2}$, Jakub Kaźmierski ${ }^{3}$, \\ and María Teresa Arredondo Waldmeyer ${ }^{1}$ \\ ${ }^{1}$ Universidad Politécnica de Madrid, ETSI Ingenieros de Telecomunicación, \\ 28002 Madrid, Spain \\ ${ }^{2}$ Grupo de investigación clínica del cerebro, cognición y conducta, \\ Consorci Sanitari de Terrassa, Barcelona, Spain \\ \{mvancells, mgarolera\}@cst.cat \\ 3 Department of Old Age Psychiatry and Psychotic Disorders, \\ Medical University of Lodz, Łódź, Poland \\ jakub.kazmierski@umed. lodz.pl
}

\begin{abstract}
Mild Cognitive Impairment (MICI) symptoms are one of the main issues that contribute, in older people, to the difficulty to live independently, social isolation and loss of autonomy. INFINITy solution provides a set of services aimed to reinforce and support the daily routines of people with MCI for both indoor and outdoor scenarios. A co-design session with end-users were performed in order to better adapt the INFINITy solution to the needs and characteristics of the target beneficiaries. Results show the feedback received form end-users regarding different aspects of the solution such as: functionalities, use cases, and interfaces. The results were useful to improve the INFINITy solution to better address user's needs and preferences.
\end{abstract}

Keywords: Co-design $\cdot$ Participatory design $\cdot$ Older users $\cdot$ Functional independence $\cdot$ Mild cognitive impairment $\cdot$ Dementia

\section{Introduction}

It is estimated that 75 million of people will live with Dementia in 2030 worldwide and this number is expected to double every 20 years, to 132 million in 2050. Due to its widespread high incidence the World Health Organization recommended that Dementia should be treated and handled as a major public health issue [1] and its prevention and treatment currently represent one of the major challenges for researches, health and social services worldwide. Impairments in memory and spatial navigation 
associated with aging and, in some cases, to a cognitive condition such as Mild Cognitive Impairment (MCI) or Dementia, are one of the main issues that contribute to the difficulty to live independently, social isolation and a progressive loss of autonomy [1]. Behavioral changes and spatial disorientation that leads to develop a wandering behavior and reduce social activities and interactions, are important early indicators of a potential evolution from normal aging to other more severe cognitive conditions [2]. Different ICT solutions could help to prevent the evolution to these conditions or maintain as long and possible a condition in which people could live independently and continue to perform their daily routines, but most of them do not provide the necessary support and tools while performing outdoor activities [3].

Based on this, the INFINITy project offers a technological solution to cover some of the needs of people with MCI, with the ambition of safeguarding their independence and autonomy while performing their daily routines for as long as possible. The main objective is to have a positive impact on the Quality of Life (QoL) of these people and their main caregiver, while preserving their functionality and extending their autonomy in indoor and outdoor activities by using a technological solution.

Currently there are two main directions collecting data from users with cognitive impairments. Either within the framework of clinical trials which often entails large cohorts of users but rarely focuses on ICT solutions but rather on the medicinal effects, or studies involving the effect of ICT solutions to people with cognitive impairments focused on cognitive impairment as co-morbidity or results because of the primary health condition. The important of QoL metrics and techniques are of vital importance for testing quality of living assessment in older people. The primary INFINITy innovation is to estimate the real-life depiction of the potentially positive effect of ICT solution on cognitive decline in both indoors and outdoors activities of daily living.

The manuscript presents the process followed to design an ICT solution together with potential end users. Section 2 presents the materials used, the sample, and methods followed to conduct the co-design sessions with end-users. Section 3 describe the design of the INFINITy solution showing the use cases defined and the first designs of the solution (i.e. interfaces and NFC tags formats) used as an initial visual material. Section 4 presents the key findings from co-design sessions regarding system designs and defined use cases. Finally, Sect. 5 presents the conclusions.

\section{Materials and Methods}

The following sub-sections present the materials used and the methods followed to carry out the co-design sessions with end-users. The findings obtained following this approach provide useful information that will be used to define and develop the final INFINITy solution.

\subsection{Materials}

The main materials used for the co-design sessions were: (1) the questionnaires used to collect user's feedbacks, both standardized and designed for the occasion questionnaires, including questions regarding social and leisure activities, technology use, 
interfaces look and feel, functionalities and use cases; (2) the interface mock-ups of the INFINITy solution; and (3) the NFC tags presented in different formats.

Technologies used for the INFINITy solution (e.g. NFC, BLE) were selected based on their low costs, easiness to use, availability and compatibility with current smartphones, and communication capabilities that fulfill the main purpose of the features provided by INFINITy.

Specifically, the standardized questionnaires used were: Socioeconomic Subjective Status (SES) [4], Montreal Cognitive Assessment (MoCa) [5], Lawton \& Brody test [6], and Memory Failures of Everyday (MFE) [7].

Consent must be obtained from all participants including the individuals who act as their informal caregiver. Participants were provided with the information they need to make an informed decision. In the occurrence of a change in the informal carer during the testbed duration, consent must be obtained from the new carer before they can become involved in the evaluation. A series of Consent Form Sheets were provided in English to all clinical partners, detailing the list of items to be consented. All participants completed the consent before the start of the co-design session.

\subsection{Sample}

The study involved 20 participants from Spain and Poland (10 participants by site) including people with MCI and their caregivers. Caregivers participated during the codesign sessions as supporters of the MCI participants as well as providing their opinions specifically in the definition of features and use cases. Demographic data collected from MCI participants is the following: age mean of 72.7 ( $\mathrm{sdv} 5.96), 50 \%$ of the sample is in the age range from 71 to 80 years old; 8 females and 2 males; schooling level with a mean of $8.4(\mathrm{sdv} 4.06)$ years of schooling; MoCa mean score of 23.9 ( $\mathrm{sdv} 2.13)$; a functionality level of 6.8 (sdv 1.03); and SES mean score of 4.7 (sdv 1.159).

\subsection{Co-design Sessions Protocol}

The co-design sessions aim to design and improve different technical aspects of the application in terms of interface design and workflow of functionalities. The session consists of an interview conducted by a project member of testbed site, where the participant and the caregiver will go through different questions related to interface design and functions of the proposed solution. The questions were defined in an easy and understandable way in order to collect the opinions and suggestions from MCI participants and their caregivers.

Specifically, the objectives of the co-design sessions were:

- Define the format and icons design of NFC tags (using questionnaires and set of NFC cards and media material)

- Assess first set of use cases and get ideas for new use cases (using questionnaires and videos of the Smart Cards)

- Get feedback regarding the mock-ups of the interface (using the mock-ups and the questionnaires) 
Each session had an approximate duration of 90 min. A written informed consent was provided to the participant and caregiver in order to be part of the co-design session and to approve video recording of the session to later visualize and analyze the answers. These recordings will only be viewed by members of the project, and subsequently deleted from the system.

Three main phases of the study were defined: (1) socio-demographic assessment; (2) psychometric assessment; and (3) qualitative assessment.

Socio-demographic Assessment: In this phase information regarding age, sex, years schooling, profession, marital status and socioeconomic status (SES questionnaire) were collected from participants.

Psychometric Assessment: The MoCa questionnaire and the Lawton \& Brody test were used to collect information about cognitive and functional state. Additionally, the Subjective Cognitive Complaints were measured using the most important items from the MFE questionnaire. The items used are the following: forgetting where you have put something; having to go back to check whether you have done something; forgetting that you were told something yesterday; and getting lost on a place where you have only been once or twice before.

Qualitative Assessment: A questionnaire designed for the occasion questions related to social and leisure activities, technology use, interfaces, functionalities and use cases was provided to participants. The project member guiding the session went through all the specific questions complementing the information provided in the questionnaire with additional materials such as screen shots of the interfaces and NFC tags in different formats.

\section{INFINITy Solution Design}

The INFINITy solution is aimed to support daily life activities of older people with MCI in indoor and outdoor environments in order to reinforce their functional abilities and safeguard their independence and autonomy while performing daily routines. It is constituted by a smartphone App (on Android) and a set of cards with the contact less technology Near-Field-Communication (NFC). In addition, a set of Bluetooth Low Energy (BLE) beacons located around the city are used to activate automatic messages while walking around.

\subsection{Use Cases}

\subsubsection{Support to Indoor and Outdoor Activities with NFC Cards}

Different use cases (UC) with NFC cards were designed in order to support daily life activities in both, indoors and outdoors scenarios. Table 1 shows the description of each defined UC. These use cases were pre-defined in order to assess and refine them during the co-designed session with MCI participants and with their caregivers. 
Table 1. Use cases with NFC cards.

\begin{tabular}{|c|c|c|}
\hline Environment & UC name & Description \\
\hline \multirow[t]{5}{*}{ Indoors } & Voice mail & $\begin{array}{l}\text { It allows a bidirectional communication between the user and } \\
\text { their caregiver by sending (recording) and receiving } \\
\text { (listening) voice messages through two paired of NFC cards }\end{array}$ \\
\hline & Battery & It provides the battery status of the smartphone \\
\hline & $\begin{array}{l}\text { Pre-fixed } \\
\text { message }\end{array}$ & It sends a predefined message to the caregiver \\
\hline & $\begin{array}{l}\text { Date and } \\
\text { time }\end{array}$ & It provides the current date and time \\
\hline & Agenda & $\begin{array}{l}\text { It allows to create an event on the agenda or consult the } \\
\text { existing ones }\end{array}$ \\
\hline \multirow[t]{4}{*}{ Outdoors } & Go home & It guides the user to go home from wherever he is \\
\hline & Go to a POI & $\begin{array}{l}\text { It guides the user to go to a specific Point of Interest from } \\
\text { wherever he is }\end{array}$ \\
\hline & $\begin{array}{l}\text { Call a } \\
\text { contact }\end{array}$ & It makes a phone call to a contact \\
\hline & $\begin{array}{l}\text { Share a } \\
\text { GPS }\end{array}$ & It sends the GPS location of the user to their caregiver \\
\hline
\end{tabular}

\subsubsection{Automatic Messages to Reinforce Abilities}

In addition to the use cases described in the previous section, there is an additional use case related to the reinforcement of the abilities through the stimulation of the declarative memory using associative principles to link two pieces of information by content and context relationships. While the user with MCI is walking around the city, the city sends them messages to their smartphone with the aim to support their daily life activities. Messages are in the form of quick games (or quizzes) and healthy or wellbeing tips and are launched every time that a user passes nearby a Point of Interest mapped with GPS or with a BLE beacon.

\subsection{Interface First Designs}

Based on the previous use cases, the INFINITy App was designed as a launcher application that enables the different functionalities of the system. It provides a simple and user-friendly interface, where the caregiver can access to set up the initial configuration of the system and where the user with MCI can access normally their standard applications installed on the phone.

\subsubsection{Main Menu (Launcher) Interface}

Different mockups of the main interface were designed to offer a variety of options for the co-design sessions: two options in portrait mode (A and B), two options in landscape mode (C and D), two options with soft colors (A and C), and two options with vivid colors ( $\mathrm{B}$ and $\mathrm{D})$. Figure 1 shows the four design options of the main menu interface. 
Portrait mode

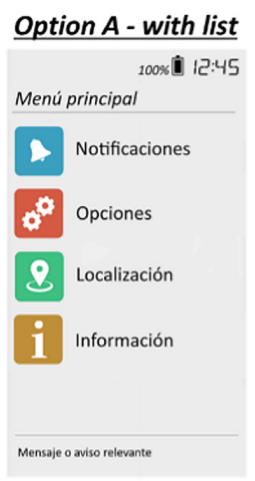

Landscape mode
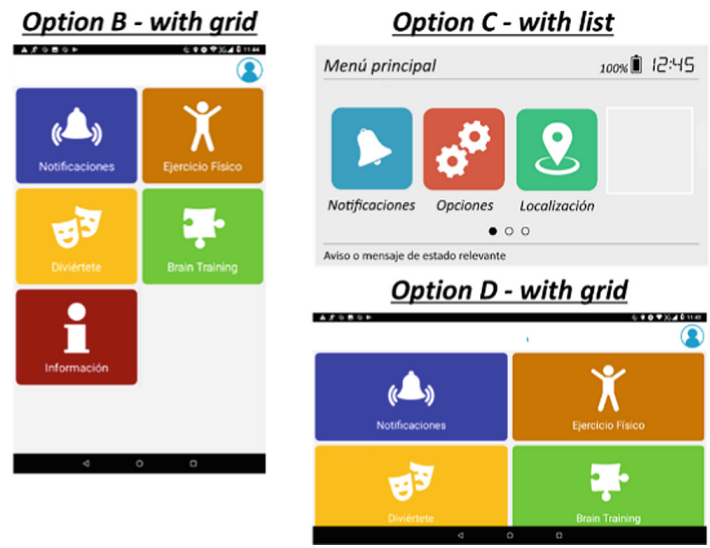

Fig. 1. Four design options for the user interface of the main menu.

In addition, two themes were designed: one with light background (A) and one with dark background (B), as presented in Fig. 2.

\section{Option A (Light Theme)}

Menú principal

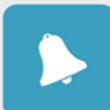

Notificaciones

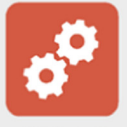

Opciones

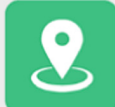

Localización

- $\circ \circ$
100\% 1.

\section{(1000}

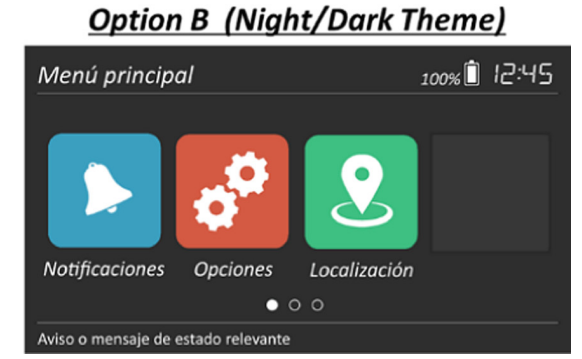

Fig. 2. Two design option with different themes.

\subsubsection{Automatic Messages Interface}

Three mockups defined to provide the messages to reinforce abilities while the user is walking around the city. The first presents message launched when a user passes nearby a region (POI) mapped with GPS or BLE in the INFINITy system. This screen informs the user that he is walking nearby a concrete type of POI and asks whether a quick quiz can be launched. The second one presents the quiz and provides the user with two options to answer the question proposed. The third provides the tip/recommendation related to the POI. 


\subsubsection{Other Interface Buttons and Functionalities}

In addition, other interface components were designed with the aim to discover what was preferred by the end users according to the font type, status bar, icons and navigation elements. Moreover, a pair of mockups were designed to show the different contrasts proposed, three with light background colors and black font, and three with dark background colors and white font.

\subsection{NFC Cards Format}

During co-design sessions it was also provided a set of options to understand which is the best format to provide the NFC cards for indoor and outdoor scenarios. Taking into account the wide offer of formats in which an NFC chip can be embedded and presented, the following six were selected as the most appropriate for the proposed use cases: credit card, key chain, pendant, ring, wristband and fridge magnetic.

\section{Key Findings from Co-design Sessions}

Before analyzing the results obtained regarding the functionalities and interface design of the solution, it is important to analyze the overall general opinion about technologies, perceived level of difficulty of smartphones and technology usage.

Regarding technological level of participants, the majority do not use smartphones (70\%). Specific responses of different statements were asked regarding technology use with a liker scale from 1 to 5 where 1 means completely disagree, 2 disagree, 3 neutral, 4 agree and 5 completely agree. Participants reported that they find smartphones hard to use (mean 3.30 SD 1.34), feel that new technologies are not for them (mean 3.50 SD 1.65) and they feel overwhelmed with so much technology (mean 3.10 SD 1.85).

Pre-defined use cases were assess by participants in order to give their opinons about their usefulness. In the case of outdoor use cases, $70 \%$ of the participants in general perceive that all use cases presented are very useful for them. Regarding, indoor use cases the $90 \%$ of the participants think that the voice mail use case is the most useful for them, followed by send pre-fixed messages, with $70 \%$, and know the battery level with a $60 \%$. The use cases that MCI participants liked the most were: phonebook management, use of calendar, know date and time, outdoors guidance, make calls and send messages.

Specifically, where asked about their opinions regarding the additional use case related to the reinforcement of the abilities through the stimulation of the declarative memory. In this respect, $50 \%$ of participants find it useful, the other half thinks that in some cases could represent an additional workload (30\%) and could be annoying $(40 \%)$. 
Results regarding the interface designs participants likes more option B (portrait mode with grid) and think it is clean, pretty and that the size of the elements are correct. In terms of screen look and feel (e.g. colors) they think that option A (portrait mode with list) colors will not tire their eyesight (70\%). Overall, $80 \%$ of participants prefer the list format although they liked more option B with a grid format, they think that having a list would be most simple to them. In the case of other interface buttons and functionalities, users prefered the options that make it more intuitive and readable, for example: icons text in plain instead of italic; navigation bars with buttons and with text; and a good contrast between background colors and font.

Futhermore, participants were asked to choose their prefered format of NFC tags for both types of use cases, indoors and outdoors. In general the most voted was the card format $(40 \%)$.

\section{Conclusions}

Co-design sessions were a fruitful experience to start involving end users from the early stages of the prototype definition, in order to better adapt the product to the target beneficiaries of the solution.

After the co-design sessions were carried out, we could conclude that a positive feedback was collected that helped us to understand the user needs and choose their preferred elements when developing the system. Feedback from end-users will be used to improve the first designs of the solutions and to identify potential new use cases and functionalities.

Acknowledgments. This work was financially supported by the EIT Health, a body of the European Union, under the activity code: 19342.

\section{References}

1. World Health Organization (WHO): Global action plan on the public health response to dementia 2017-2025 (2017). ISBN 978-92-4-151348-7

2. Alzheimer's Association: 2018 Alzheimer's disease facts and figures. Alzheimer's \& Dementia 14, no. 3, pp. 367-429 (2018). https://doi.org/10.1016/j.jalz.2018.02.001

3. Teipel, S., Babiloni, C., Hoey, J., Kaye, J., Kirste, T., Burmeister, O.K.: Information and communication technology solutions for outdoor navigation in dementia. Alzheimer's Dementia 12(6), 695-707 (2016). https://doi.org/10.1016/j.jalz.2015.11.003

4. Adler, N.E., Epel, E.S., Castellazzo, G., Ickovics, J.R.: Relationship of subjective and objective social status with psychological and physiological functioning: preliminary data in healthy, White women. Health Psychol. 19(6), 586 (2000). https://doi.org/10.1037/02786133.19.6.586

5. Montreal Cognitive Assessment (MoCa). Spanish version. https://www.mocatest.org/pdf_ files/test/MoCA-Test-Spanish.pdf. Polish version https://www.mocatest.org/pdf_files/test/ MoCA-Test-Polish.pdf 
6. Lawton, M.P., Brody, E.M.: Assessment of older people: self-maintaining and instrumental activities of daily living. Gerontologist 9(3), 179-186 (1969)

7. Montejo, P., Peña, M., Sueiro, M.: The memory failures of everyday questionnaire (MFE): internal consistency and reliability. Spanish J. Psychol. 15, 768-776 (2012). https://doi.org/ 10.5209/rev_SJOP.2012.v15.n2.38888

Open Access This chapter is licensed under the terms of the Creative Commons Attribution 4.0 International License (http://creativecommons.org/licenses/by/4.0/), which permits use, sharing, adaptation, distribution and reproduction in any medium or format, as long as you give appropriate credit to the original author(s) and the source, provide a link to the Creative Commons license and indicate if changes were made.

The images or other third party material in this chapter are included in the chapter's Creative Commons license, unless indicated otherwise in a credit line to the material. If material is not included in the chapter's Creative Commons license and your intended use is not permitted by statutory regulation or exceeds the permitted use, you will need to obtain permission directly from the copyright holder.

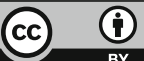

